\title{
A quantitative method for calculating irradiation area of laser target designator
}

\author{
ZHANG Jiandong", HUANG Zhiyi, and SHI Guoqing \\ School of Electronics and Information, Northwestern Polytechnical University, Xi' an 710072, China
}

\begin{abstract}
In laser-guided bomb attacking process, the target indication from the laser target designator is the premise for the bomb to hit the target accurately. Considering the lack of quantitative study of the irradiation area of the laser target designator, this paper, based on the existing aircraft motion model and the laser transmission model, uses two aircraft as respectively the carrier of the laser-guided bomb and the carrier of the laser designator and proposes a method to calculate the global irradiation area of the airborne laser designator. By using the proposed algorithm, the global irradiation area when attacking a large flat target or a large spherical target is simulated respectively. Finally, according to the simulation results, the influences of different factors on the shapes of the irradiation area are discussed in detail.
\end{abstract}

Keywords: laser-guided bomb, irradiation area, laser target designator, semi-active guidance.

DOI: $10.21629 / J S E E .2019 .04 .01$

\section{Introduction}

At present, semi-active laser guidance [1,2] has been widely used in air-to-ground attack missions, and semiactive laser-guided bombs [3], due to their characteristics of high precision, high efficiency [4,5] and low cost [6], become one of the commonly used guided weapons in military operations.

A successful operational use of the semi-active laserguided bomb needs the cooperation of the semi-active laser seeker which is implemented in the guided bomb and the laser target designator which is carried by an aircraft. When the designator illuminates a ground target in a certain spatial area, the seeker on the bomb can receive the reflected laser beam from the target and thus control the fins and fly towards the target.

Currently, there are mainly two frequently-used types of attacking modes of laser-guided bombs [4]: self attacks

\footnotetext{
Manuscript received June 04, 2018.

*Corresponding author.

This work was supported by the Aeronautical Science Foundation of China (ASFC-20135153031; ASFC-20135553035; ASFC-2017ZC53033).
}

and cooperative attacks.

In self attacks, the aircraft, by using the airborne laser target designator, will act as the illuminator for own carried laser-guided bombs.

In cooperative attacks, however, the operational task is accomplished by two aircraft. In this situation, the attacking process of the laser-guided bomb is generally performed by an aircraft equipped with a laser-guided bomb (bomber aircraft) and an aircraft equipped with a laser target designator (designator aircraft) $[7,8]$. When the attack starts, the designator aircraft will irradiate the target firstly, and then, the bomber aircraft will release the bomb. After releasing the bomb, the bomber aircraft retreats from the battlefield and the designator aircraft continues to irradiate the target until the bomb hits the target [9]. Since compared with the ground target designator [10], there is less terrain limitation to the airborne target designator [11] and the bomber aircraft can immediately retreat from the battlefield to avoid being attacked by the enemy. The dual aircraft cooperative attacking mode becomes a hotspot of current military research.

To the best knowledge of us, until now, most research on laser-guided bombs is mainly confined to two aspects: (i) analyzing the influences of different factors on laser transmission process [12,13]; (ii) calculating the launch acceptable region of the bombs [9] or the capture zones of the guided bombs [14].

In [9], an improved fast pattern search algorithm was developed to search the boundary of the launch acceptable region within fewer iterations. In [14], based on the instant capture area model, the total capture area of laser guided bomb was obtained. In [12], according to the laser transmission model, the influence of jamming measures was analyzed in detail. In [13], the impacts of various atmospheric factors on the transmission of $1.06 \mu \mathrm{m}$ laser were studied in detail.

By reviewing the published articles, we notice that in 
the aspect of operational configuration, the principles of how to deploy the laser target designator after releasing laser-guided bombs are mostly described qualitatively, and the lack of a quantitative description of the irradiation area makes the problem of how to irradiate targets and guide bombs more obscure. However, a quantitatively described guideline is of critical importance to the practical use of guided weapons. To this end, this paper takes into consideration the atmospheric impact on laser transmission, and proposes a quantitative method to obtain the irradiation area of the designator aircraft in the laser-guided bomb attacking process. The proposed method can make the aforementioned problem clearer and become a guideline for future deployment of laser target designators.

\section{Definition of irradiation area of designator aircraft or laser target designator}

In the laser-guided bomb air-to-ground attacking process, the space relationship of the designator aircraft, the laserguided bomb and the ground target is shown in Fig. 1.

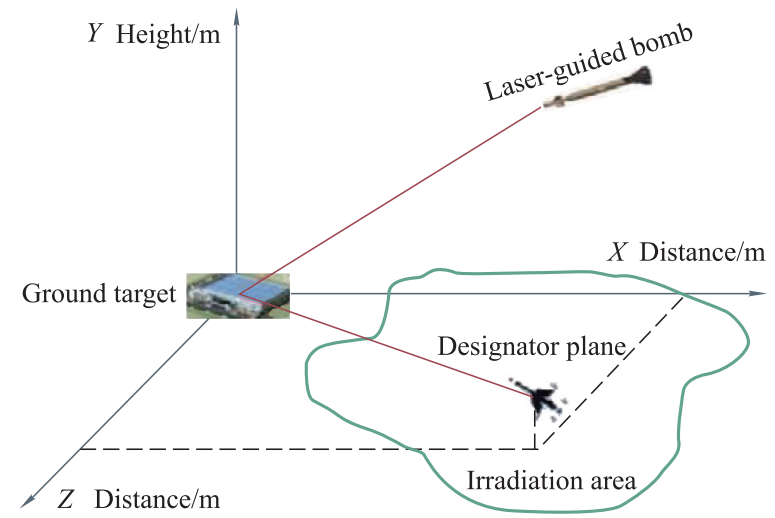

Fig. 1 Illustration of synergistic attacking process

In fact, the reflected laser received by the laser seeker of the bomb can be effectively detected only when it reaches a certain energy density [15], and the minimum value of the energy density is called the energy density threshold.

In order to ensure that guided bombs capture the target's location, the reflected laser received by the laser seeker needs to reach its energy density threshold. Since laser transmission is influenced by propagation distance and angle [13,16-18], the airborne laser target designator of the designator aircraft needs to be confined to a certain spatial area and keeps on irradiating the ground target. Otherwise the seeker cannot receive sufficient reflected laser energy to identify the ground target.

In this paper, this spatial area is defined as the irradiation area of the designator aircraft/laser target designator, referred to as the irradiation area in the following part of the paper.

\section{Modeling for laser-guided bomb and ground target}

\subsection{Laser-guided bomb motion model}

Assume that the guided bomb is a simple laser-guided bomb which is thrust-free, axisymmetric, low-resistance and non-rotating. Ignore the impact of air resistance on the motion of the bomb.

In the geographic coordinate system (taking north as the direction of $x$-axis, up-vertical as the direction of $y$ axis and the direction of $z$-axis is determined by the righthand rule), the coordinate of the bomb is $(x, y, z)$, and a three-degree-of-freedom motion model $[19,20]$ can be established as follows:

$$
\left\{\begin{array}{l}
\frac{\mathrm{d} v}{\mathrm{~d} t}=-g \sin \alpha \\
\frac{\mathrm{d} \alpha}{\mathrm{d} t}=\frac{g}{v}(n d-\cos \alpha) \\
\frac{\mathrm{d} \beta}{\mathrm{d} t}=-\frac{g \cdot n t}{v \cos \alpha} \\
\frac{\mathrm{d} x}{\mathrm{~d} t}=v \cos \alpha \cos \beta \\
\frac{\mathrm{d} y}{\mathrm{~d} t}=v \sin \alpha \\
\frac{\mathrm{d} z}{\mathrm{~d} t}=v \cos \alpha \sin \beta
\end{array}\right.
$$

where $v$ is the bomb scalar speed, $\alpha$ is the ballistic tilt angle, $\beta$ is the ballistic deflection angle, $g$ is the gravity acceleration, $n d$ and $n t$ are respectively the components of the normal overload in the vertical plane and the horizontal plane.

The control law of the laser-guided bomb adopts proportional navigation [21-24]. Since the bomb is non-rotating, its motion can be decomposed into the movement in the vertical plane and the movement in the horizontal plane. Here we take the overload $n d$ and $n t$ as control variables:

$$
\left\{\begin{array}{l}
n d=\frac{k d \cdot v}{g} \times q d+\cos \alpha \\
n t=-\frac{k d \cdot v \cdot \cos \alpha}{g} \times q t
\end{array}\right.
$$

where $k d$ is the scale coefficient of the proportional navigation method, $q d$ is the angle between the coordinate axis and the projection of the bomb-target line on the $x O y$ plane and $q t$ is the angle between the coordinate axis and the projection of the bomb-target line on the $x \mathrm{O} z$ plane.

\subsection{Ground target model}

This paper focuses on the case of the laser-guided bomb attacking the large, stationary target on the ground. When an incident laser beam irradiates diffuse reflectors that have different geometric shapes, there will be a great difference 
in the intensity distribution of the reflected laser. Therefore, according to the difference in the shape of the ground targets, the targets are mainly divided into two categories: large flat targets and large spherical targets, which will be discussed below separately.

\subsubsection{Large flat targets}

In fact, the top of the enemy's important military facilities, such as the military factory and the military base, basically has a flat structure and belongs to this category. This paper assumes the surface area of this kind of ground targets is no less than $50 \mathrm{~m}^{2}\left(S \geqslant 50 \mathrm{~m}^{2}\right)$.

\subsubsection{Large spherical targets}

Apart from the aforementioned case, some important enemy production facilities, such as chemical storage tanks, and the tops of some defense facilities have spherical shapes. These targets are also the focus in air-to-ground combat operations. We also assume that the surface area of this kind of spherical targets is no less than $50 \mathrm{~m}^{2}(S \geqslant$ $50 \mathrm{~m}^{2}$ ).

\section{Laser transmission model}

\subsection{Target reflection model}

In the air-to-ground attacking process, if the reflected laser is within a wide reflection angle, its energy intensity is approximately consistent with the Lambert cosine law [16]. Therefore, within the allowable range of error, this paper considers the reflection surface of ground targets as an ideal Lambert surface, following the Lambert cosine law.

$P_{0}$ is the power of the laser beam applied on the Lambert surface, and $\varphi$ is the reflection angle.

When a certain amount of laser is applied to a Lambert surface, as shown in Fig. 2, the intensity of the reflected light can be calculated [25] as

$$
I_{\theta}=\frac{\rho \cdot P_{0}}{\pi} \cdot \cos \varphi
$$

where $I_{\theta}$ is the intensity of the reflected light in the direction of $\varphi$, and $\rho$ is the reflectivity of the Lambert surface.

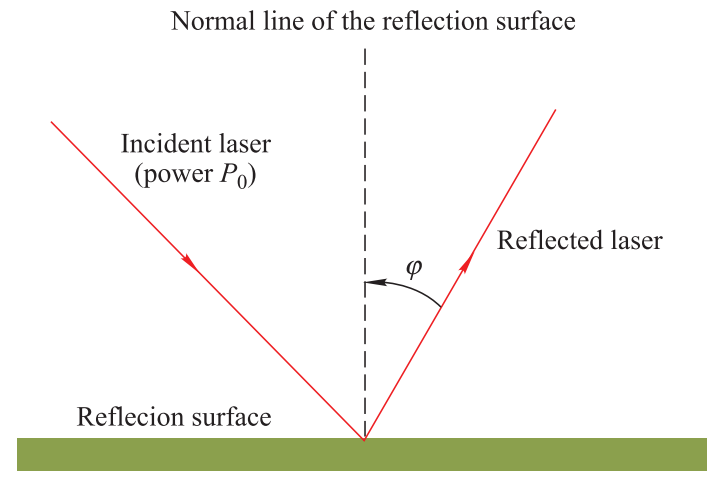

Fig. 2 Illustration of Lambert's cosine law

\subsection{Laser atmospheric transmission model}

In this paper, the wavelength of the laser generated by the laser target designator is assumed to be $1.064 \mu \mathrm{m}$ [4].

The influence of atmosphere on laser transmission is mainly reflected in three aspects: atmospheric attenuation effect, atmospheric backscatter effect and atmospheric turbulence effect. In order to simplify the research, this paper assumes that the atmosphere is uniform and considers the atmospheric attenuation effect on the laser transmission process.

The attenuation effect on the laser consists of two aspects: the attenuation by atmospheric molecules and the attenuation by atmospheric aerosols. Among them, the attenuation by atmospheric molecules to $1.064 \mu \mathrm{m}$ laser is negligible, and the attenuation by atmospheric aerosols plays a major role [25].

The laser target designator and the laser seeker of the laser-guided bomb both have a height difference with the ground target. Namely, there is a certain height difference between the two ends of the optical path. Therefore, the attenuation of the laser can be calculated by using the atmospheric transmittance on an oblique path. For a $1.064 \mu \mathrm{m}$ laser beam, in the geographical coordinate system, the atmospheric transmittance on an oblique path $\tau$ is calculated as follows:

$$
\tau=\exp \left\{-\frac{2.06}{0.83 R_{V} \sin \alpha_{T}}\left(1-\mathrm{e}^{-0.83 R_{p} \cos \alpha_{T}}\right)\right\}
$$

where $\tau$ is the atmospheric transmittance on an oblique path, $R_{v}$ is the atmospheric visibility, also known as the meteorological distance, $R_{p}$ is the propagation distance of laser, and $\alpha_{T}$ is the zenith angle of the designator or the laser seeker $[13,16]$.

\subsection{Reflected laser energy received by the seeker}

When the laser target designator irradiates a large ground target, it can be considered that all the laser spot on the ground falls on the target reflection surface, and the area of the laser spot is thus equal to the area of target diffuse reflection.

Assume that $\omega$ is the divergence angle of the laser beam and $R$ is the incident optical path length. Since $\omega$ is very small (about $0.5 \mathrm{mrad}$ in general cases), it can be considered $\tan \omega=\omega$. Therefore, the area of target diffuse reflection can be obtained as follows [26]:

$$
A_{0}=\pi\left(R \tan \frac{\omega}{2}\right)^{2} \approx \frac{1}{4} \pi R^{2} \omega^{2} .
$$

During the laser-guided bomb attacking process, the space relationship of the target designator, the ground target and the bomb is shown in Fig. 3 . 


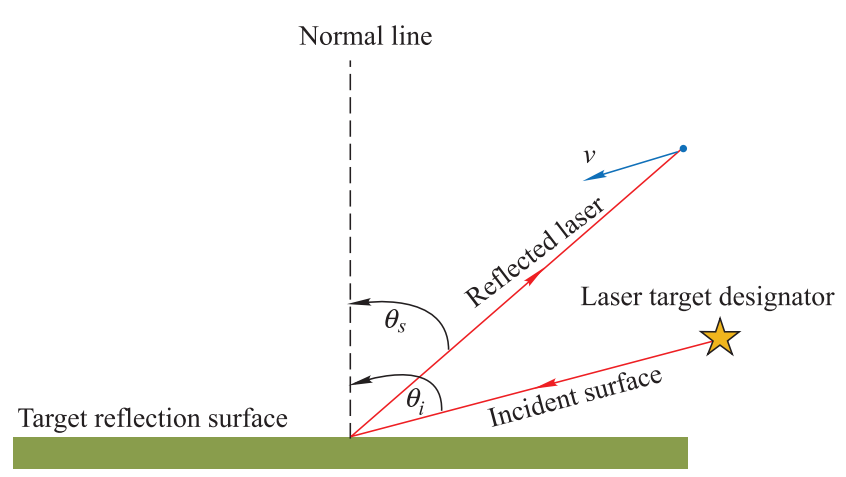

Fig. 3 Illustration of received energy model

$v$ is the velocity of the laser-guided bomb, $\theta_{i}$ is the incidence angle and $\theta_{s}$ is the reflection angle.

The relationship between the laser energy emitted by the target designator and the laser energy received by the laser seeker can be calculated as follows [12]:

$$
U_{t}=\frac{U_{0} T_{t} \tau_{1} \tau_{2} \rho T_{r} A_{s} \cos \varepsilon \cos \theta_{i} \cos \theta_{s}}{\pi R_{2}^{2}}
$$

where $U_{t}$ is the laser energy received by the laser seeker, $U_{0}$ is the pulsed laser beam energy emitted by the laser target designator, $T_{t}$ is the optical transmittance of the target designator, $\tau_{1}$ is the atmospheric transmittance on the incident path $R_{1}, \tau_{2}$ is the atmospheric transmittance on the reflection path $R_{2}, T_{r}$ is the optical transmittance of the laser seeker, $A_{s}$ is the energy receiving area of the seeker, and $\varepsilon$ is the angle between the seeker's optical axis and the normal line of the diffuse reflection surface.

According to (6), the laser energy density $U$ received by the seeker can be obtained as follows:

$$
U=\frac{U_{t}}{A_{s}}=\frac{U_{0} T_{t} \tau_{1} \tau_{2} \rho T_{r} \cos \varepsilon \cos \theta_{i} \cos \theta_{s}}{\pi R_{2}^{2}} .
$$

\section{Algorithm for calculating irradiation area of laser target designator}

In this paper, the shape of the irradiation area is determined by two factors: large target requirement and the constraint of energy density threshold.

\subsection{Basic assumptions}

Optical transmittances of the target designator and the seeker are equal to $1\left(T_{t}, T_{r}=1\right)$. Since they are constants between 0 and 1 , this simplification does not change the shape of the irradiation field.

Given that the seeker's capture angle is generally small (about $10^{\circ}-15^{\circ}$ ) and the curvature of laser-guided bomb trajectory is small, it can be approximated that $\cos \varepsilon \approx 1$.
The laser target designator is aimed at the geometric center of the target all the time when irradiating the ground target.

\subsection{Large flat target situation}

\subsubsection{Large target constraint}

Under the basic assumptions, in order to ensure the whole laser spot falls on the target surface, according to (5), (8) should be satisfied:

$$
\frac{1}{4} \pi R^{2} \omega^{2} \leqslant \frac{1}{10} S
$$

where $S \geqslant 50 \mathrm{~m}^{2}$ refers to the surface area of the ground target. Therefore, the following constraints can be obtained:

$$
\left\{\begin{array}{l}
R \leqslant \sqrt{\frac{4 \times 50 m^{2}}{10 \pi \times \omega^{2}}} \\
y_{1} \geqslant 0 \\
R_{\max }=\sqrt{\frac{4 \times 50 m^{2}}{10 \pi \times \omega^{2}}}
\end{array}\right.
$$

where $R$ is the length of the incident optical path, and $R_{\max }$ is the maximum value of $R$.

\subsubsection{The constraint of energy density threshold}

According to (4), the atmospheric transmittance on an oblique path is rewritten as

$$
\tau=\exp \left\{-\frac{2.06 R_{p}}{0.83 R_{v} \sqrt{R_{p}^{2}-H^{2}}}\left(1-\mathrm{e}^{-0.83 H}\right)\right\}
$$

where $H$ refers to the height of the laser target designator and the laser guided bomb.

In the geographic coordinate system, assume that the ground target is located at the origin of the coordinate system and the bomb trajectory is in the $x O y$ plane. The coordinates of the target designator are $\left(x_{1}, y_{1}, z_{1}\right)$ and the coordinates of the bomb are $\left(x_{2}, y_{2}, z_{2}\right)$. Therefore, $\tau_{1}, \tau_{2}$, $\cos \theta_{i}$ and $\cos \theta_{s}$ can be calculated as follows:

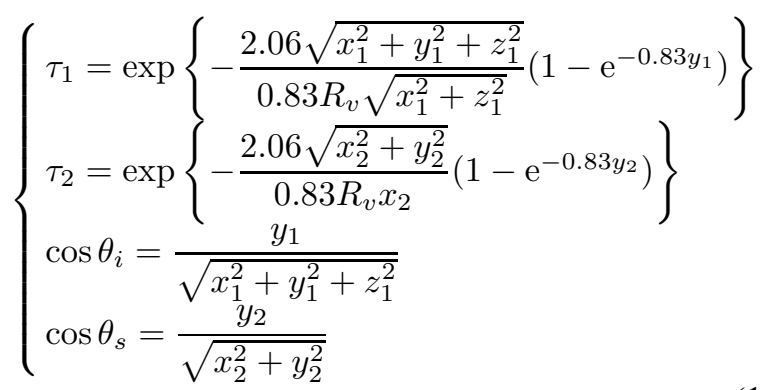

where $\tau_{1}$ and $\tau_{2}$ are the atmospheric transmittance on the incident path and the atmospheric transmittance on the reflection path respectively, $\theta_{i}$ is the incidence angle (the angle between the incident laser and the normal line of the 
target reflection surface), and $\theta_{s}$ is the reflection angle (the angle between the reflected laser and the normal line of the target reflection surface).

Substituting these parameters into (7), we obtain the laser energy density $U$ received by the seeker.

$$
\begin{gathered}
U=\frac{U_{0} T_{t} \tau_{1} \tau_{2} \rho T_{r} \cos \varepsilon \cos \theta_{i} \cos \theta_{s}}{\pi R_{2}^{2}}= \\
\frac{U_{0} \rho}{\pi R_{2}^{2}} \cdot \mathrm{e}^{-\frac{2.06 \sqrt{x_{2}^{2}+y_{2}^{2}}\left(1-\mathrm{e}^{-0.83 y_{2}}\right)}{0.83 R_{v} \cdot x_{2}}} \cdot \mathrm{e}^{-\frac{2.06 \sqrt{x_{1}^{2}+y_{1}^{2}+z_{1}^{2}}\left(1-\mathrm{e}^{-0.83 y_{1}}\right)}{0.83 R_{v} \sqrt{x_{1}^{2}+z_{1}^{2}}}} . \\
\frac{y_{2}}{\sqrt{x_{2}^{2}+y_{2}^{2}}} \cdot \frac{y_{1}}{\sqrt{x_{1}^{2}+y_{1}^{2}+z_{1}^{2}}}
\end{gathered}
$$

Considering the time relationship between the position of the bomb and the position of the target designator, during the falling process of the bomb, the irradiation area of the designator varies from time to time. However, due to various factors (terrain limits, air defense weapons, etc.), the designator aircraft which carries the target designator cannot meet the requirement of the rapid changing irradiation area. Therefore, it is the global irradiation area that has practical value, an area which meets the energy density threshold constraint all the time in the whole attacking process.

Rewrite (12) as follows:

$$
U=L \times\left(\mathrm{e}^{-\frac{2.06 \sqrt{x_{1}^{2}+y_{1}^{2}+z_{1}^{2}}\left(1-\mathrm{e}^{-0.83 y_{1}}\right)}{0.83 R_{v} \sqrt{x_{1}^{2}+z_{1}^{2}}}} \cdot \frac{y_{1}}{\sqrt{x_{1}^{2}+y_{1}^{2}+z_{1}^{2}}}\right) .
$$

In (13), notice that the formula in bracket only contains the parameters of the laser target designator.

Simulate bomb trajectory by using (1) and (2) and then, according to the trajectory parameters at different time points, get the minimum value $L$ of the rest part in (12). Here $L$ indicates the worst impact of the other factors on the energy density received by the seeker, and the energy density threshold constraint is obtained.

$$
\left\{\begin{array}{l}
U_{\min } \leqslant L \times\left(\mathrm{e}^{-\frac{2.06 \sqrt{x_{1}^{2}+y_{1}^{2}+z_{1}^{2}}\left(1-\mathrm{e}^{-0.83 y_{1}}\right)}{0.83 R_{v} \sqrt{x_{1}^{2}+z_{1}^{2}}}} .\right. \\
\quad \frac{y_{1}}{\sqrt{x_{1}^{2}+y_{1}^{2}+z_{1}^{2}}} \\
y_{1} \geqslant 0
\end{array}\right.
$$

Combine the constraints in Sections 5.2.1 and 5.2.2 together, the coordinate set that meets the constraints in (9) and (14), forms the global irradiation area of the laser target designator.

\subsection{Large spherical target}

\subsubsection{Large target constraint}

The requirement for the irradiation field in this situation is the same as it is in the large flat target situation.

\subsubsection{The constraint of energy density threshold}

In this situation, the space relationship is shown in Fig. 4.

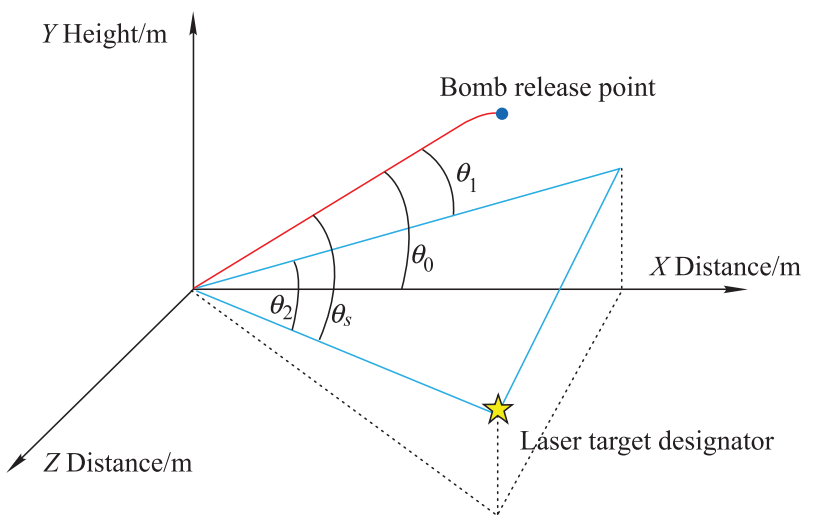

Fig. 4 Space relationship in laser-guided bomb attack process

$\theta_{1}$ is the angle between the projection of the designatortarget line on the $x O y$ plane and the bomb-target line, $\theta_{2}$ is the angle between the designator-target line and the $x O y$ plane, and $\theta_{s}$ is the reflection angle (in this situation, it is equivalent to the angle between the incident laser and the reflected laser received by the bomb, as shown in Fig. 4).

Since the designator is aimed at the center of the target sphere, the incidence angle $\theta_{i}$ (namely the angle between the incident laser and the normal line of the reflection surface) is zero $\left(\cos \theta_{i}=1\right)$ and the reflection angle $\theta_{s}$ is equal to the angle between the incident laser and the reflected laser. According to the simulated ballistic parameters, in the bomb falling process, the bomb-target line tilt angle $\theta$ (the angle between the bomb-target line and the ground) changes very few (please refer to Table 1 ). Therefore, we use a constant value $\theta_{0}$ (the value of $\theta$ at the moment of bomb release) to replace $\theta$.

The values of angles shown in Fig. 4 can be obtained as follows.

$$
\left\{\begin{array}{l}
\cos \theta_{s}=\cos \theta_{1} \cos \theta_{2} \\
\cos \theta_{1}=\frac{x_{1} \cos \theta_{0}+y_{1} \sin \theta_{0}}{\sqrt{x_{1}^{2}+y_{1}^{2}}} \\
\cos \theta_{2}=\frac{\sqrt{x_{1}^{2}+y_{1}^{2}}}{\sqrt{x_{1}^{2}+y_{1}^{2}+z_{1}^{2}}}
\end{array}\right.
$$

Substitute the parameters into (7) and obtain

$$
\begin{aligned}
& U=\frac{U_{0} \rho}{\pi R_{2}^{2}} \cdot \frac{x_{1} \cos \theta_{0}+y_{1} \sin \theta_{0}}{\sqrt{x_{1}^{2}+y_{1}^{2}+z_{1}^{2}}} . \\
& \mathrm{e}^{-\frac{2.06 \sqrt{x_{2}^{2}+y_{2}^{2}}\left(1-\mathrm{e}^{\left.-0.83 y_{2}\right)}\right.}{0.83 R_{v} \cdot x_{2}}} . \\
& \mathrm{e}^{-\frac{2.06 \sqrt{x_{1}^{2}+y_{1}^{2}+z_{1}^{2}}\left(1-\mathrm{e}^{-0.83 y_{1}}\right)}{0.83 R_{v} \sqrt{x_{1}^{2}+z_{1}^{2}}}} .
\end{aligned}
$$


Follow the similar idea as discussed in Section 5.2.2, extract the target designator part from (16) and use $L$ to represent the worst impact of the other factors on the energy density received by the seeker. The energy density threshold constraint is obtained.

$$
\left\{\begin{array}{l}
U_{\min } \leqslant L \times\left(\mathrm{e}^{-\frac{2.06 \sqrt{x_{1}^{2}+y_{1}^{2}+z_{1}^{2}}\left(1-\mathrm{e}^{-0.83 y_{1}}\right)}{0.83 R_{V} \sqrt{x_{1}^{2}+z_{1}^{2}}}}\right. \\
\left.\quad \frac{x_{1} \cos \theta_{0}+y_{1} \sin \theta_{0}}{\sqrt{x_{1}^{2}+y_{1}^{2}+z_{1}^{2}}}\right) \\
y_{1} \geqslant 0
\end{array} .\right.
$$

Combine the constraints in Sections 5.3.1 and 5.3.2. Similarly, the coordinate set that meets the constraints in (9) and (17), forms the global irradiation area of the laser target designator.

\section{Simulation results and analysis}

\subsection{Trajectory simulation}

Assume that the ground target is located at the origin of the coordinate system, the initial conditions are as follows: the bomb-release coordinate is $(5000,0,2$ 000), the initial speed of the bomb is $200 \mathrm{~m} / \mathrm{s}$, the initial trajectory of the bomb tilt angle is $0^{\circ}$, the initial trajectory deflection angle of the bomb is $180^{\circ}$, the scale coefficient $k d$ is 10 , the pulse energy of the laser target designator is $130 \mathrm{~mJ}$ $[8,27]$, the reflectivity $\rho$ of the target surface is 0.5 , and the atmospheric visibility $R_{v}$ is $20 \mathrm{~km}$. According to the bomb motion model, the bomb trajectory parameters can be obtained.

Simulation results are listed below, as shown in Fig. 5, Fig. 6 and Table 1.

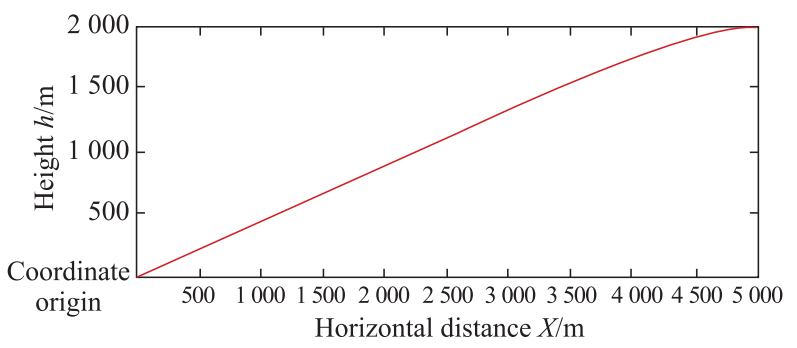

Fig. 5 Trajectory simulation of laser-guided bomb

Bomb ballistic parameters are listed in Table 1.

Table 1 Ballistic parameters

\begin{tabular}{cc}
\hline Type & Value \\
\hline Landing speed $V / \mathrm{m} \cdot \mathrm{s}^{-1}$ & 344.09 \\
Landing ballistic tilt angle $\gamma /\left(^{\circ}\right)$ & -14.35 \\
Range of $\theta /\left(^{\circ}\right)$ & $-24.22-(-21.80)$ \\
Range of $\cos \theta$ & $0.912-0.929$ \\
Flight time $t / \mathrm{s}$ & 40.95 \\
Landing error $d / \mathrm{m}$ & 1.53 \\
Value of $L$ (flat surface) & $2.41 \times 10^{-10}$ \\
Value of $L$ (spherical surface) & $6.50 \times 10^{-10}$ \\
\hline
\end{tabular}

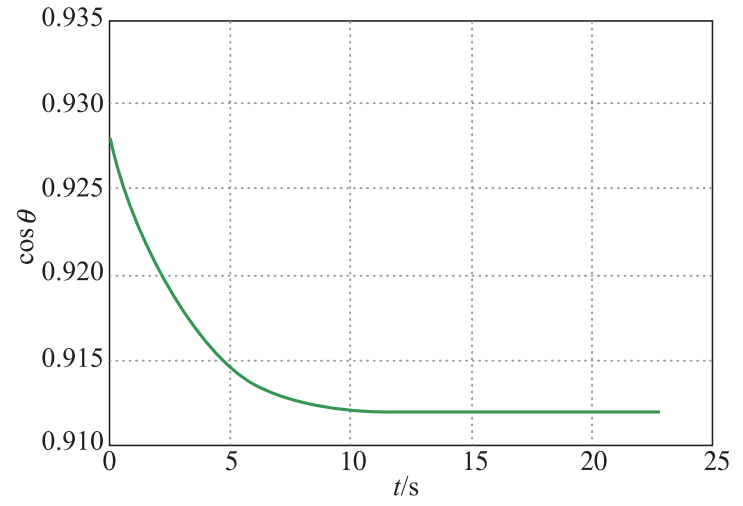

Fig. 6 Range of $\cos \theta$ values

According to the simulation results, the landing error is $1.53 \mathrm{~m}$, and this tolerable result verifies the validity of the guidance law and the motion model. From the range of $\theta\left(-24.22^{\circ}-\left(-21.80^{\circ}\right)\right)$ and the range of $\theta(0.912-0.929)$, it can be seen $\theta$ changes very little in the bomb's falling process.

\subsection{Simulation for large flat target situation}

Based on the constraints proposed in Section 4.2 and the value of $L$ (flat surface) in Section 5.1, the irradiation area in the large flat target situation is obtained, as shown in Fig. 7 and Fig. 8.
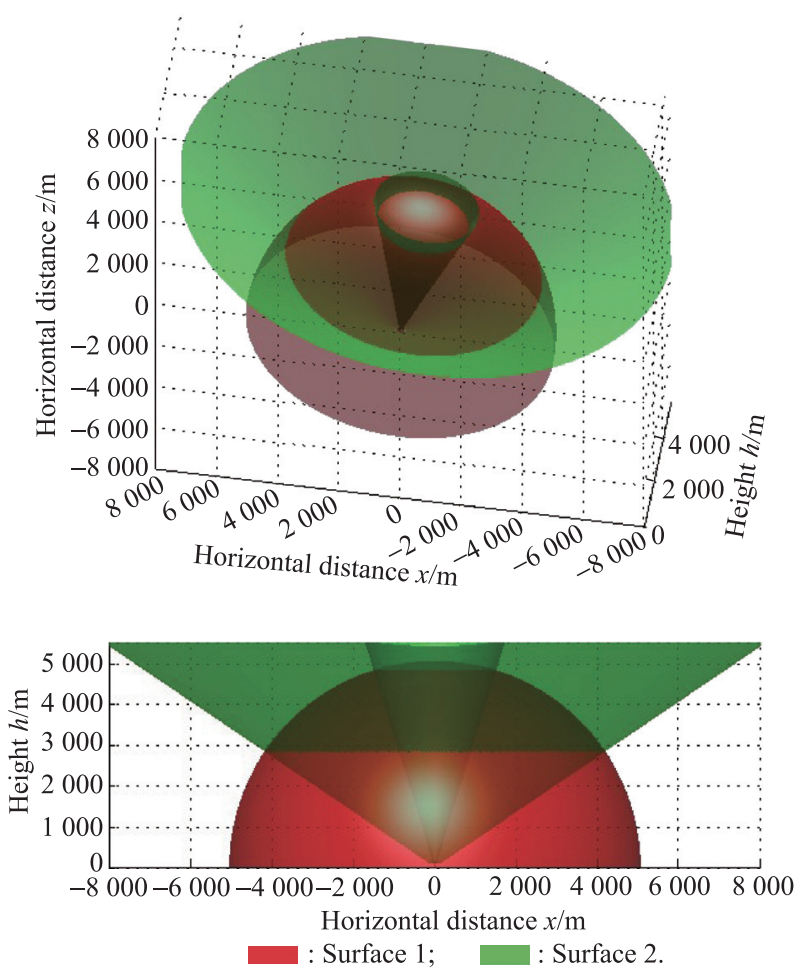

Fig. 7 Simulation result of large flat target irradiation field (low sensitivity) 

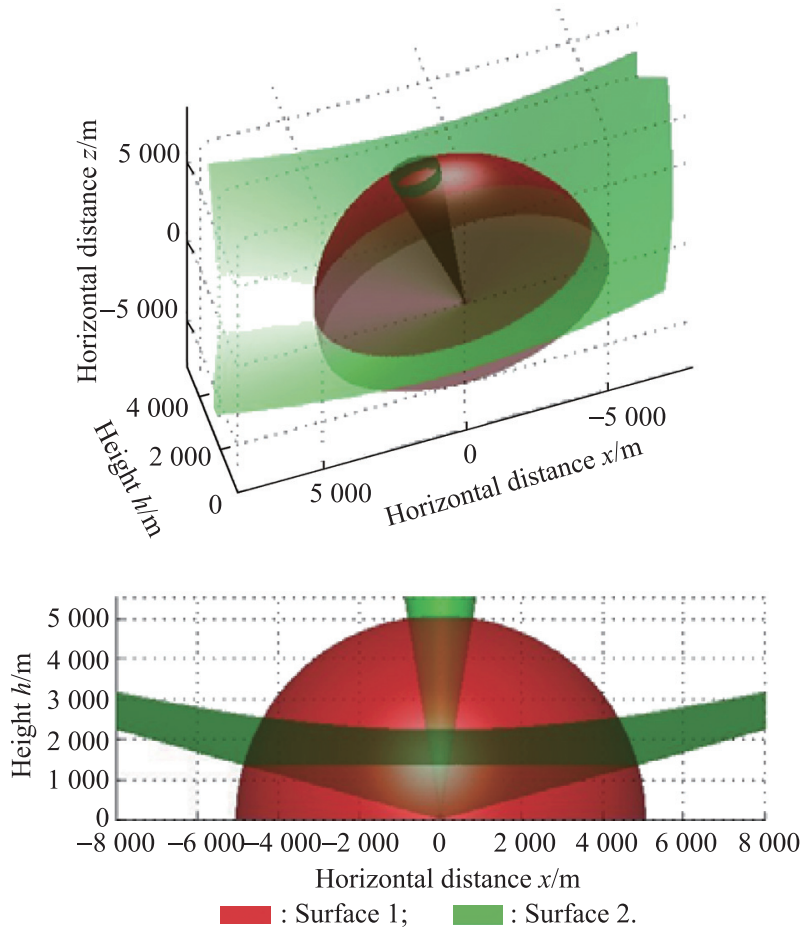

Fig. 8 Simulation result of large flat target irradiation field (high sensitivity)

(i) When the energy density threshold of the seeker is high

The global irradiation area is the space enclosed by Surface 1 and Surface 2. In the bomb falling process, if the target designator keeps irradiating the target within the global irradiation area, the requirement of the energy density threshold will be satisfied all the time.

Surface 1 is the result of a large target constraint. Surface 2 is the result of the constraint of the energy density threshold. Discuss Surface 2 in detail.

The function of Surface 2 is shown in (18).

$$
U_{\min }=L \times\left(\mathrm{e}^{-\frac{2.06}{0.83 R V \sin \alpha_{T}}\left(1-\mathrm{e}^{-0.83 R \cos \alpha_{T}}\right)} \cdot \cos \theta_{i}\right)
$$

It can be considered that Surface 2 is the combination of two surfaces: inner surface and outer surface.

Inner surface: when the target designator crosses the inner surface and approaches $y$-axis, $\theta_{i}$ and $\alpha_{T}$ approach zero. The changing rates of $\cos \theta_{i}$ and $\cos \alpha_{T}$ approach zero accordingly and the values of $\cos \theta_{i}$ and $\cos \alpha_{T}$ change little; however, the changing rate of $\sin \alpha_{T}$ is big and the value of $\sin \alpha_{T}$ approaches zero rapidly, which causes the exponent value in (18) to decrease, and thus the energy density received by the seeker no longer meets the threshold requirement. Here, the exponent part in (18) plays a major role. That is, the atmospheric attenuation effect is the main reason for forming the inner surface.
Outer surface: when the target designator crosses the outer surface and becomes farther from $y$-axis, the values of $\theta_{i}$ and $\alpha_{T}$ increase. The changing rate of $\cos \theta_{i}$ is big and the value of $\cos \theta_{i}$ approaches zero rapidly. Here, $\cos \theta_{i}$ in (18) plays a major role. That is, the target diffuse reflection is the main reason for forming the outer surface.

(ii) When the energy density threshold of the seeker is low

By comparing Fig. 7 and Fig. 8, it can be seen that as the energy density threshold decreases (namely, detection performance increases), the outer surface is close to the $x \mathrm{Oz}$ plane and the inner surface is closer to $y$-axis, which indicates that the constraint of the energy density threshold is relaxed and the designator aircraft can fly in a larger spatial area when designating the target.

\subsection{Simulation for large spherical target situation}

Based on the constraints proposed in Section 4.3 and the value of $L$ (spherical surface) in Section 5.1, the irradiation area in the large spherical target situation is obtained, as shown in Fig. 9 and Fig. 10.

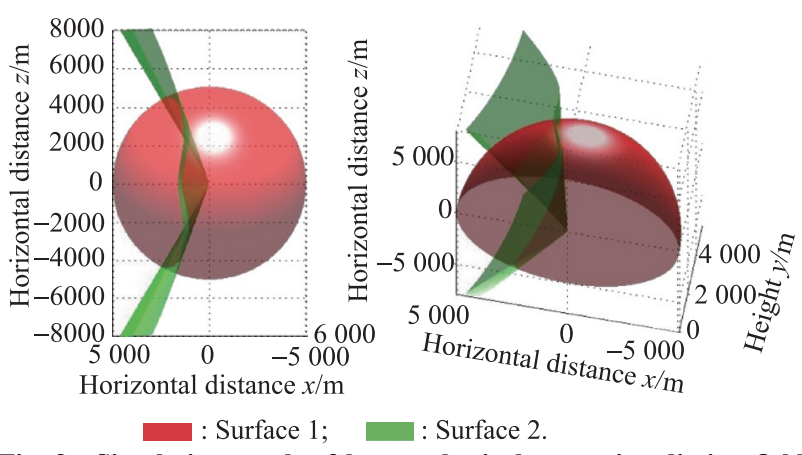

Fig. 9 Simulation result of large spherical target irradiation field (low sensitivity)

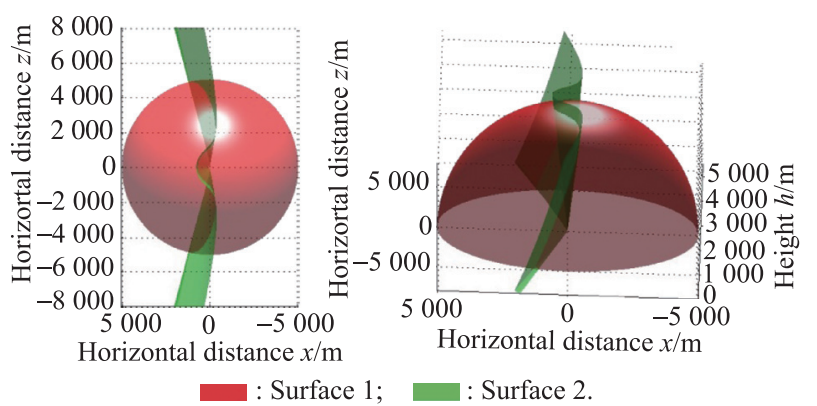

Fig. 10 Simulation result of large spherical target irradiation field (high sensitivity)

(i) When the energy density threshold of the seeker is high

The global irradiation area is the space enclosed by Surface 1 and Surface 2. Similarly, Surface 1 is caused by a large target constraint and Surface 2 is caused by the constraint of the energy density threshold. 
(ii) When the energy density threshold of the seeker is low

With regard to Surface 2, consider Surface 2 as the combination of two surfaces. The function of Surface 2 is shown in (19).

$$
U_{\min } \leqslant L \times\left(\mathrm{e}^{-\frac{2.06}{0.83 R_{V} \sin \alpha_{T}}\left(1-\mathrm{e}^{-0.83 R \cos \alpha_{T}}\right)} \cdot \cos \theta_{s}\right) .
$$

Here we divide (19) into two parts: the exponent part and $\cos \theta_{s}$, where the exponent part represents the influence of the atmospheric attenuation effect and $\cos \theta_{s}$ represents the influence of the target diffuse reflection. As the energy density threshold decreases, an obvious depression appears in the middle of Surface 2. This depression is caused by the atmospheric attenuation effect (resemble the inner surface in a large flat situation). As for the other part of Surface 2, when the target designator crosses it, $\cos \theta_{s}$ will decrease rapidly, which indicates the target diffuse reflection is the main reason for this part of Surface 2 .

(iii) Changes in the shape of Surface 2 under different values of $U_{\min }$

Looking into the details of Surface 2, according to Fig. 11, the shape of Surface 2 varies with values of $U_{\min }$ $\left(3 \mathrm{fJ} / \mathrm{cm}^{2}, 10 \mathrm{fJ} / \mathrm{cm}^{2}, 30 \mathrm{fJ} / \mathrm{cm}^{2}\right)$. By comparison, as $U_{\min }$ decreases, Surface 2 splits into two parts (the red one in Fig. 11). As discussed in (ii), these two parts are mainly caused by the atmospheric attenuation effect and the target diffuse reflection respectively.

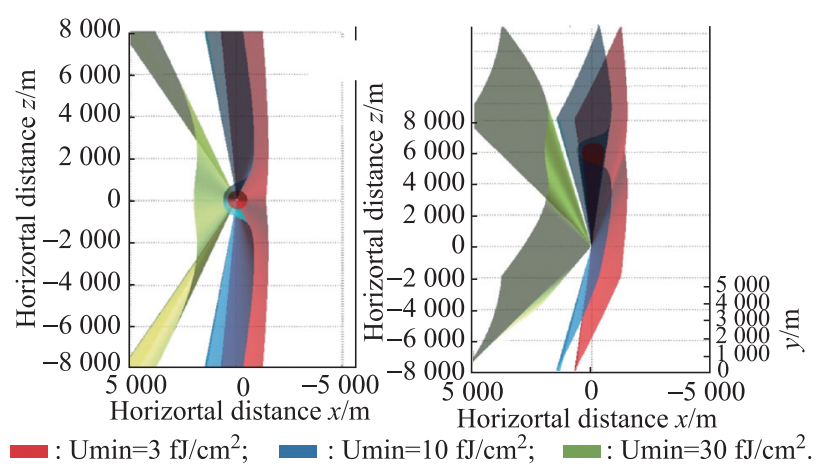

Fig. 11 Changes in the shape of Surface 2

In conclusion, from the simulation result (Figs. 7-11), the decrease of the seeker's energy density threshold can enlarge the global irradiation field of the laser target designator. By comparing the simulation results of the irradiation field in Sections 5.2 and 5.3, it can be seen that the shape of the target reflection surface will greatly affect the shape and size of the global irradiation field.

\section{Conclusions}

During the attacking process of the laser-guided bomb, the laser designator irradiating the target correctly is the prerequisite for the bomb accurately hitting the target. Based on the motion model and the laser transmission model, this paper puts forward an algorithm for quantitatively calculating the global irradiation area of the laser target designator, which provides a quantitative algorithm for the application of the airborne laser target designator, and has certain theoretical significance. Simulation results indicate the shape and size of the irradiation area change with the shape of the ground target and the energy density threshold of the bomb seeker.

Due to the complexity of the laser transmission process, to build a more precise model, atmospheric scattering and turbulence shall be put into consideration in future work.

\section{References}

[1] HUBBARD K, KATULKA G, LYON D, et al. Low-cost semiactive laser seekers for US army application. Proc. of the International Telemetering Conference, 2008: 27 - 30 .

[2] ALLEN R T. COTS lens and detector characterization for low cost miniature SAL seekers. Proc. of the AIAA Guidance, Navigation \& Control, 2013: 5199-5205.

[3] ANDERBERG B, WOLBARSHT M L. Laser weapons: the dawn of a new military age. New York: Springer, 2013.

[4] KAUSHAL H, KADDOUM G. Applications of lasers for tactical military operations. IEEE Access, 2017, 5: 20736-20753.

[5] FANG Y C, GUO L H, LI Y, et al. Jamming effectiveness analysis of the weather vane type laser guided bombs by laser. Chinese Journal of Luminescence, 2013, 34(5): 656-664. (in Chinese)

[6] WEI C, HAN Y, CUI N, et al. Fifth-degree cubature Kalman filter estimation of seeker line-of-sight rate using augmenteddimensional model. Journal of Guidance, Control, and Dynamics, 2017, 40(9): 2352-2359.

[7] SHARMA M. Evaluation system for laser target designators. Journal of Battlefield Technology, 2010, 13(1): 35-40.

[8] SABATINI R, RICHARDSON M A, GARDI A, et al. Airborne laser sensors and integrated systems. Progress in Aerospace Sciences, 2015, 79: 15-63.

[9] ZHANG Y, WANG N, CHEN J, et al. Research on launch acceptable region for guided bombs in air-to-ground multitarget attack. Acta Armamentarii, 2011, 32(12): $1474-1480$. (in Chinese)

[10] LEE S T, SILVER M, BARRON A, et al. A compact laser target designator. Proc. of the Laser Technology for Defense and Security XII, 2016, 9834: 98340Q.

[11] DONZELLI P, MAROZZA R. Laser designation pod on the Italian air force AM-X aircraft: a prototype integration. Proc. of the NATO/RTO SCI Joint Symposium on Advances in Vehicle Systems Concepts and Integration, 1999: A16.1-A16.10.

[12] ZHANG W P, WU J H, HU X, et al. A test method to real laser seekers based on electro-optic countermeasure. Infrared and Laser Engineering, 2013, 42(3): 637-642. (in Chinese)

[13] LIU W C, QI L L, HE H R, et al. Numerical study on atmospheric transmission of $1.06 \mu \mathrm{m}$ laser. Laser \& Infrared, 2011, 41(5): 520-524. (in Chinese)

[14] ZHANG D C, XIA Q L, HE X F. Rapid calculation method for capture area of laser guided bomb. Infrared and Laser Engineering, 2015, 44(12): 3535-3540. (in Chinese)

[15] CLEARFIELD W H, CAPEHART B L. Stochastic optimization of some of the design parameters of airborne laser-seeker 
systems. IEEE Trans. on Aerospace and Electronic Systems, 1972, AES-8(1): 19-26.

[16] EVSIKOVA L G, GOREMYKIN Y A. Determining the atmospheric transmittance for laser wavelengths on an oblique track. Journal of Optical Technology, 2016, 83(8): 486-489.

[17] WANG Y, LIU Y, LI H, et al. The research of atmospheric transmittance of high power laser along a ground-space slant path. Proc. of the 5th International Symposium on Photoelectronic Detection and Imaging: High Power Lasers and Applications, 2013: 89040B.

[18] MENG J Q. Research on field test method of laser seeker sensitivity threshold. Aero Weaponry, 2000, 5: 14-16, 18. (in Chinese)

[19] CARRETERO J G, NIETO F J S, CORDÓN R R. Aircraft trajectory simulator using a three degrees of freedom aircraft point mass model. Ad Hoc Networks, 2013, 13(1): 191-209.

[20] SUDARS M. Development and simulations of aircraft guidance system using a 3 -degree-of-freedom point mass model. Aviation, 2009, 13(2): $35-43$.

[21] JEON I S, LEE J I, TAHK M J. Homing guidance law for cooperative attack of multiple missiles. Journal of Guidance Control and Dynamics, 2010, 33(1): 275-280.

[22] ULYBYSHEV Y R. Terminal guidance law based on proportional navigation. Journal of Guidance, Control, and Dynamics, 2005, 28(4): $821-824$

[23] RALPH J F, EDWARDS K L. The effect of aircraft biases on the delivery of an enhanced laser-guided weapon. ICAS Paper, 2002: 511.1-511.9.

[24] MAC T T, COPOT C, KEYSER R D, et al. The development of an autonomous navigation system with optimal control of a UAV in partly unknown indoor environment. Mechatronics, 2018, 49: $187-196$.

[25] JIA X J. Optimization of laser semi-active guidance parameters. Nanjing, China: Nanjing University of Science and Technology, 2004. (in Chinese)

[26] SABATINI R, RICHARDSON M A, GARDI A, et al. Airborne laser sensors and integrated systems. Progress in Aerospace Sciences, 2015, 79: 15-63.

[27] COFFEY D W, NORRIS V J. YAG: $\mathrm{Nd}^{\wedge}+3$ laser target desig- nators and range finders. Applied Optics, 1972, 11(5): 1013 1018.

\section{Biographies}

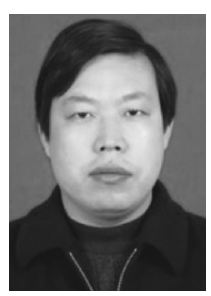

ZHANG Jiandong was born in 1974. He is an associate professor at the Department of System and Control Engineering in Northwestern Polytechnical University, China. He received both his M.S. and $\mathrm{Ph} . \mathrm{D}$. degrees in systems engineering from the same university. His research fields and interests include modeling of complex weapon system, effectiveness evaluation of complex systems and advanced fire control theory.

E-mail: jdzhang@nwpu.edu.cn

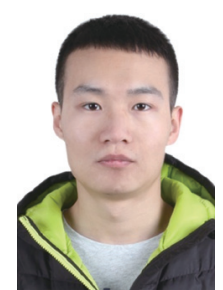

HUANG Zhiyi was born in 1994. He received his bachelor degree in Northwestern Polytechnical University, Xi'an, in 2016. Since 2016, he has been a postgraduate student majoring in systems engineering in Northwestern Polytechnical University. His main research interests are modeling of guided weapons, multi-UAVs target assignment and its application on control and decision of UAVs.

E-mail: zhiyi.huang@ hotmail.com

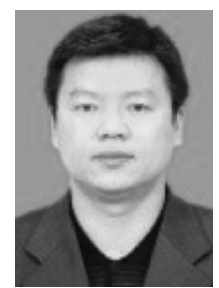

SHI Guoqing was born in 1974. He is an associate professor at the Department of Systems and Control Engineering in Northwestern Polytechnical University, China. He received his M.S. and Ph.D. degrees in systems engineering from the same university. His research fields and interests include modeling and simulation of aeronautical weapon system, integrated avionics system measurement \& test technologies, development and design of embedded real-time systems, modeling simulation and effectiveness evaluation of complex systems.

E-mail: Shiguoqing@nwpu.edu.cn 